\title{
On the importance of partial frequency redistribution in modeling the scattering polarization
}

\author{
K. N. Nagendra \\ Indian Institute of Astrophysics, Koramangala, Bengaluru 560 034, India \\ email: knn@iiap.res.in
}

\begin{abstract}
It is well-known that partial frequency redistribution (PRD) is the basic physical mechanism to correctly describe radiative transfer in spectral lines. In the case of polarized line scattering, the PRD becomes particularly important to describe the line-wing polarization, instead of the well-known mechanism of complete redistribution (CRD). Historically, the twolevel atom PRD scattering matrices for polarized line scattering were first derived in the 1970's, and later generalized to the case of arbitrary fields in 1997. The latter formulation of the PRD matrices have subsequently been used in the solution of the line transfer equation to successfully model the non-magnetic (resonance scattering) and the magnetic (Hanle scattering) polarization observations. In recent years, using the Kramers-Heisenberg approach, we formulated PRD matrices for various physical mechanisms like quantum interference involving fine- and hyperfinestructure states in a two-term atom. The effect of collisions is included in an approximate way. We have used these PRD matrices to model the observed linear polarization in several interesting lines of the Second Solar Spectrum. In this paper I present a few results which highlight the importance of PRD in the interpretation of the polarized Stokes profiles.
\end{abstract}

Keywords. Polarization, radiative transfer, scattering, stars: atmospheres, Sun: chromosphere

\section{Introduction}

It is well-known that the angular anisotropy of the solar/ stellar radiation within the atmosphere is essential to produce linear polarization upon scattering on the bound states of atoms and molecules. The angular anisotropy (limb darkening/ limb brightening) naturally exists due to the source function gradient that prevails in stellar atmospheres. In the non-magnetic case, unequal populations of the magnetic substates created by the incidence of anisotropic radiation on the scattering atom produce the polarization of the scattered radiation. When the initial and final states are identical, the polarization is produced by 'resonance (Rayleigh) scattering'. When they are different, the polarization is produced by 'Raman scattering'. In both cases, the frequency non-coherence in the scattering event arises purely due to the random motion of the atoms (i.e. the mechanism of 'frequency redistribution' by Doppler effect). The collisions involving radiating and colliding atoms/ electrons causes the so called collisional frequency redistribution.

The degeneracy of magnetic substates is lifted in the presence of an external magnetic field. When the magnetic splitting is much smaller than the fine or hyperfine structure splitting, the line polarization is governed by the linear Hanle-Zeeman effect - the limit in which the magnetic energy shift depends linearly on the field strength. When the magnetic splitting is comparable or larger than the fine or hyperfine structure splitting, the line polarization is described by the Paschen-Back effect. The well-known Hanle effect represents the modification of the resonance scattering polarization due to the presence of a magnetic field. 
In this paper we consider the scattering polarization in a two-level or a two-term atom controlled by frequency redistribution processes. We neglect the lower level or lower term polarization. It is well established that partial frequency redistribution (PRD) correctly describes the scattering in spectral lines. Thus, it is necessary to use PRD when calculating line polarization in strong lines formed under NLTE. The redistribution matrix derived in Bommier (1997a, 1997b) represents the most sophisticated expression established to describe scattering polarization in a two-level atom in the presence of arbitrary magnetic fields (see also Bommier \& Stenflo 1999, Sampoorna et al. 2007a, 2007b, Sampoorna 2011). More recently Bommier (2014) formulated the multi-level atom PRD theory for scattering polarization in lines. An alternative theory based on the concept of metalevels was developed by Landi Degl'Innocenti et al. (1997) to derive collisionless redistribution matrices for scattering in multi-level atoms in the presence of magnetic fields. A heuristic approach to the problem of scattering polarization in a multi-level atom with PRD and a weak magnetic field was presented in Sampoorna et al. (2013).

Using the Kramers-Heisenberg scattering amplitude approach of Stenflo (1994), Smitha et al. $(2011 \mathrm{a}, 2013 \mathrm{a})$ formulated a PRD matrix for $J$-state interference in a two-term atom. This PRD matrix was derived in the limit of the linear-Zeeman regime (in which the magnetic splitting is much smaller than the fine structure splitting). The non-magnetic PRD matrix for the case of a two-level atom with hyperfine structure splitting was derived in Smitha et al. (2012a). This derivation accounts for $F$-state interference effects. More recently Sowmya et al. $(2014 \mathrm{a}, 2014 \mathrm{~b})$ derived the PRD matrices for $F$-state and $J$-state interference in the presence of fields with arbitrary strengths (including the Paschen-Back effect regime). In all the above-mentioned papers the lower level or lower term polarization is neglected. Recently, Casini et al. (2014) derived a generalized frequency redistribution function for the polarized two-term atom in an arbitrary magnetic field. More recently, Stenflo (2015) presented an extension of the quantum mechanical scattering theory based on the Kramers-Heisenberg approach to treat polarized scattering in multi-level atomic systems.

\section{Modeling the Second Solar Spectrum}

The linearly polarized spectrum of the Sun is referred to as the Second Solar Spectrum (Stenflo \& Keller 1997). The magnetic modification to this spectrum through the Hanle effect serves as a diagnostic tool to detect weak fields on the Sun. Here we describe a standard procedure that we adopt for modeling the Second Solar Spectrum. It involves the use of realistic models of the solar atmosphere (e.g., Fontenla et al. 1993), and realistic atomic models.

\subsection{The modeling procedure}

The theoretical polarized spectrum is calculated using a two stage process (see e.g., Holzreuter et al. 2005, Anusha et al. 2011). In the first stage, the multi-level PRD unpolarized radiative transfer problem is solved. For this purpose we use the MALI (Multi-level Approximate Lambda Iteration) code of Uitenbroek (2001, hereafter called the RH-code). This code solves the statistical equilibrium equations and the unpolarized transfer equation self-consistently and iteratively. The model atmosphere and the model atom are the two inputs to this code. The RH-code computes highly accurate and realistic specific intensity and opacities, which form the back-bone of the polarized solution computed in the second stage. The opacities are kept fixed in the second stage, and the Stokes vector $\boldsymbol{I}=(I, Q, U)^{\mathrm{T}}$ is computed perturbatively by solving the polarized transfer equation. 


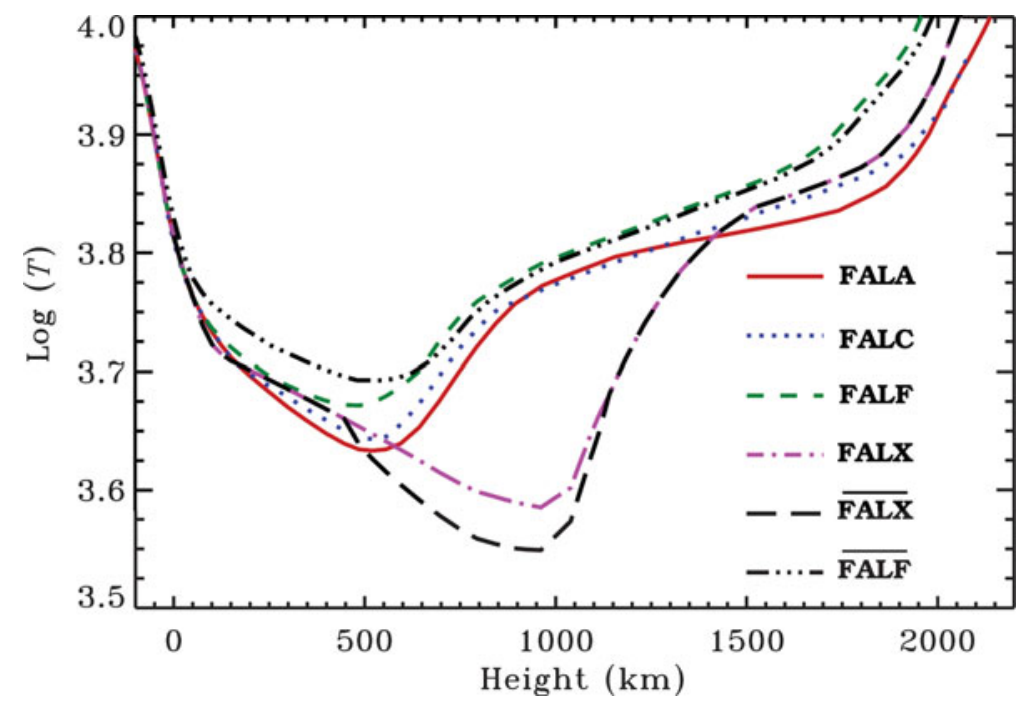

Figure 1. Temperature structure of several standard 1D model atmospheres of the Sun. $\overline{\text { FALF }}$ and FALX are models with a slightly modified temperature structure.

For simplicity, in the second stage a two-level or a two-term atomic model is assumed, depending on the problem at hand. The reference direction for positive $Q$ is defined to be parallel to the nearest solar limb.

Figure 1 shows the height dependence of the temperature for different model atmospheres that we used in our modeling work. Among this set of model atmospheres, FALX represents the coolest model, while FALF is the hottest. The $\overline{\text { FALF }}$ and FALX models were constructed modifying slightly the temperature structure over certain height ranges, in order to achieve a finer fit to the $Q / I$ profiles of the Cr I triplet and Ba II $\mathrm{D}_{2}$ line, respectively. We have verified that these modifications do not significantly affect the intensity spectrum. Like the original models, $\overline{\text { FALF }}$ and $\overline{\text { FALX }}$ continue to fit the observed center-to-limb variation of the continuum intensity for all the wavelengths covering the entire spectrum (see Smitha et al. 2012b, 2013b).

\subsection{The Hanle Effect in the Second Solar Spectrum}

It is well known that the interference between the magnetic substates $m$ of a given $J$-state is the cause of the Hanle effect in spectral lines. In this section we present an example of the Hanle effect signatures in the Second Solar Spectrum of the Ca I $4227 \AA$ line. This line exhibits the largest degree of polarization in the optical region and is formed in the lower chromosphere.

Limb observations (representing a nearly $90^{\circ}$ scattering) have been the standard mode of observing the scattering polarization, since the non-magnetic polarization reaches a maximum there. For a long time, scattering polarization near the disk-center was thought to be too small to be of practical utility due to symmetry reasons. It was first pointed out by Trujillo Buneo (2001) that the presence of a non-radial field gives rise to significant scattering polarization signatures near the disk-center via the so-called forward scattering Hanle effect. Indeed, Trujillo Bueno et al. (2002) showed through observations and modeling that it is in fact a powerful diagnostic tool to detect the weak fields near the solar disk-center. Using this forward scattering Hanle effect together with the longitudinal Zeeman effect, Anusha et al. (2011) proposed a modeling strategy to uniquely determine the vector magnetic field near the disk center. More recently, Carlin \& Asensio Ramos 

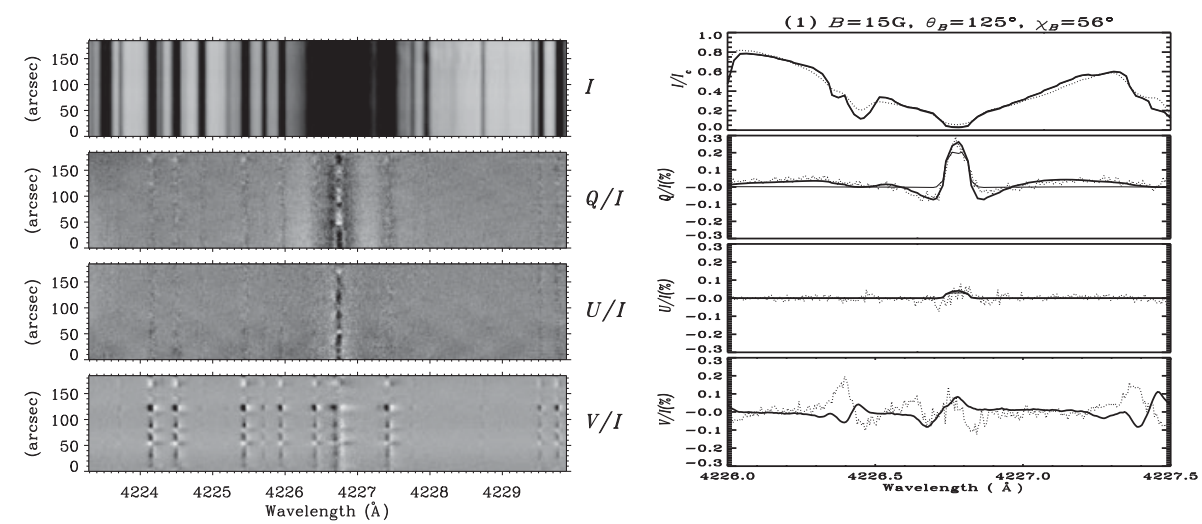

Figure 2. Left Panel: CCD image of the Stokes parameters in a spectral window around the Ca I $4227 \AA$ line. The recording was made on October 15, 2010 near the active solar region NOAA 1112. Right Panel: Illustration of the best fit of the theoretical model profiles (solid lines) to the observed profiles (dotted lines) for a given location near the disk center $(\mu=0.9)$. The thin solid lines represent the profiles computed under the assumption of CRD. This calculation used the FALC model atmosphere.

(2015) explored the diagnostic potential of forward scattering Hanle effect in the Ca II IR triplet lines considering also realistic MHD simulations.

The left panel of Figure 2 shows the CCD image of the Stokes parameters in a spectral window around the Ca I $4227 \AA$ line. These observations were performed at IRSOL (Switzerland) using the ZIMPOL-3 polarimeter. For more details on the observations see Bianda et al. (2011). Eight different positions along the slit of the spectrograph were selected. The values of the Stokes parameters corresponding to each location were obtained by averaging over 3-4 pixels. At each location the magnetic field is assumed to be uniform inside the atmosphere, with a unity filling factor.

The observed $V / I$ signals indicate the presence of weak longitudinal magnetic flux. The assumption of a unity filling factor allows us to use the "weak field approximation" (see e.g., Stenflo 1994, Landi Degl'Innocenti \& Landolfi 2004) of the Zeeman effect to model the observed $V / I$ profiles, and thereby determine the longitudinal component of the magnetic flux density. Since only the longitudinal field component is constrained in this way, we have an infinite family of possible solutions (ambiguities) for the field vector itself. When $\mu \neq 1$, these ambiguities can be eliminated by using the observed linear polarization $(Q / I$ and $U / I)$ profiles as additional constraints.

It can be shown that the transverse Zeeman effect does not contribute to the linear polarization since it would require excessively strong transverse fields. In addition, it cannot account for the shapes of the observed $Q / I$ and $U / I$ profiles (see Anusha et al. 2011 for more details). Thus, the observed $Q / I$ and $U / I$ are analyzed by solving the polarized transfer equation for the forward scattering Hanle effect including PRD. The solutions at the line center wavelength are used to construct polarization diagrams of $Q / I$ versus $U / I$. The observed line center data are placed over these polarization diagrams to extract a single vector magnetic field for which the observed and theoretical $Q / I$ and $U / I$ amplitudes agree. Several combinations of the magnetic field parameters $B, \theta_{B}$, and $\chi_{B}$ can reproduce the observed data, but only the set that is consistent with the longitudinal component obtained from the $V / I$ profile represents the correct value of $\boldsymbol{B}$. The right panel of Fig. 2 shows an example of the best fit to the observations of Stokes profiles at one location. Here we also compare the theoretical $I, Q / I$, and $U / I$ Hanle profiles computed using CRD (thin solid line) and PRD (thick solid line). Clearly, the shape and 

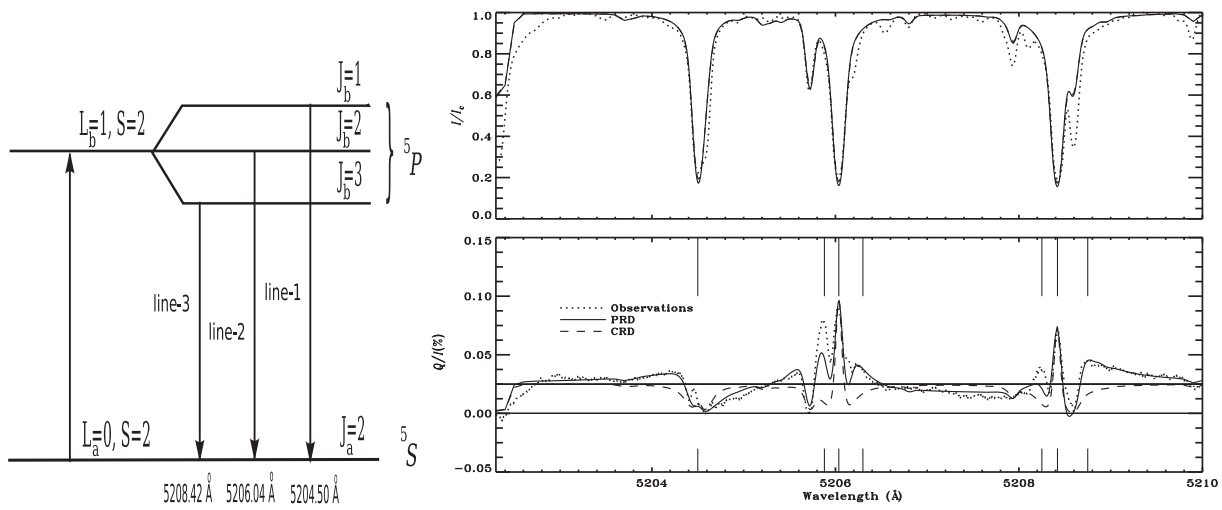

Figure 3. Left Panel: Schematic term diagram showing the transitions in the Cr I triplet. Right Panel: Illustration of the best fit of the theoretical model profiles (thick solid lines) to the observed profiles (dotted lines) for $\mu=0.15$. The two thin solid lines in $Q / I$ represent the continuum and zero polarization levels. The dashed line is the theoretical $Q / I$ computed with the approximation of CRD. The $\overline{\mathrm{FALF}}$ model atmosphere was used for the calculation. A spectral smearing of $80 \mathrm{~m} \AA$ was applied to the theoretical profiles to simulate the observations.

magnitude of the wing polarization and line center peak amplitude cannot be reproduced using the CRD mechanism. It is worth noting that this is a strong chromospheric line, and hence PRD is essential to model it.

\subsection{Quantum Interference Phenomena in the Second Solar Spectrum}

The Second Solar Spectrum exhibits signatures of a number of physical processes that are not seen in the intensity spectrum. The quantum interference among the fine or hyperfine structure energy levels of an atom is one of such physical processes whose signatures are imprinted only in the linearly polarized spectrum. For example, Stenflo (1980) showed for the first time that the scattering polarization signatures that he observed for the solar $\mathrm{Ca} I \mathrm{I} \mathrm{H}$ and $\mathrm{K}$ lines could only be explained when the $J$-state interference between the $J=1 / 2$ and the $J=3 / 2$ levels was taken into account. Other well-known examples that present signatures governed by the $J$-state interference are $\mathrm{NaI}_{\mathrm{I}} \mathrm{D}_{1}$ and $\mathrm{D}_{2}$, the $\mathrm{Mg}$ II $\mathrm{h}$ and $\mathrm{k}$ lines, and the $\mathrm{Cr}$ I triplet. When an atom possesses nuclear spin the $J$ states are split into $F$ states (hyperfine structure splitting). The quantum interference between the $F$ states produces depolarization in the line core. Examples of lines governed by $F$-state interference are $\mathrm{NaI} \mathrm{D}_{2}$, BaII $\mathrm{D}_{2}$, and the Sc II line at $4247 \AA$. In the following subsections we present our recent efforts to model lines governed by $J$ or $F$-state interference including PRD.

\subsubsection{An Example of J-state Interference Phenomena: CrI triplet at 5204, 5206, and $5208 \AA$}

The Cr I triplet arises from the $L_{a}=0 \rightarrow L_{b}=1 \rightarrow L_{a}=0$ scattering transition, where $L$ denotes the orbital angular momentum of the lower $(a)$ and the upper $(b)$ terms. The electron spin is $S=2$. Due to the $L-S$ coupling, the upper term splits into three fine structure states of total angular momentum quantum numbers $J_{b}=1,2,3$ while the lower term has only one fine structure state with $J_{a}=2$ (see left panel of Figure 3). Smitha et al. (2012b) demonstrated the importance of including PRD and the quantum interference between the upper term fine structure levels to model the observed linear polarization profiles of the Cr I triplet lines. 
The right panel of Fig. 3 shows the theoretical fit to the observed $Q / I$ spectra of these triplet lines. The observations were recorded on September 6, 2011 at IRSOL using the ZIMPOL-3 polarimeter, at the heliographic north pole with the slit placed parallel to the limb at $\mu=0.15$. In the multi-level RH-code (first stage) we use a Cr I model atom with 14 levels, 11 line transitions, and 13 continuum transitions (see Smitha et al. $2012 \mathrm{~b}$ for more details). For computing the theoretical $Q / I$ profile (second stage), we use the two-term model atom shown in the left panel of Figure 3. The $J$-state PRD matrix with angle-averaged PRD functions derived in Smitha et al. (2011a, 2013a) was used. We assume the lower term to be infinitely sharp and unpolarized. Hyperfine splitting is neglected because the most abundant (90\%) isotope of Cr I has zero nuclear spin. The effect of isotropic inelastic collisional coupling between the upper and lower fine structure levels is accounted for in the PRD matrix. However the effects of collisional coupling between the fine structure levels of the upper term are neglected. Furthermore, the depolarizing elastic collisions that couple $m$-states belonging to a given fine structure level $J_{b}$, are considered and taken to be the same for a given term. Consistently with these assumptions, we use the branching ratios corresponding to a two-level atom derived by Bommier (1997b).

The observed $Q / I$ spectra of the Cr I triplet exhibit (i) the triple peak structure in 5206 and $5208 \AA$ lines, and (ii) the cross overs between the line components about the continuum polarization level (which is characteristic of $J$-state interference). These aspects in the observed $Q / I$ profile could be reproduced only when (i) PRD effects, (ii) collisional redistribution, and (iii) the $J$-state interference between the upper fine structure states were taken into account. Clearly, PRD is the correct mechanism to be used to calculate the linear polarization profiles of strong lines. To obtain the observed line center amplitudes of $Q / I$ we used micro-turbulent magnetic fields (4 to $6 \mathrm{G}$ ) with an isotropic angular distribution (Stenflo 1994). Furthermore, a small modification of the original temperature structure of FALF (see the dash-triple-dotted line in Fig. 1) becomes necessary to obtain a good fit to the far wing region of the three lines in $Q / I$. The intensity spectrum is insensitive to the temperature structure modification, while the $Q / I$ spectrum is quite sensitive to such changes. Thus, the $Q / I$ spectrum serves as an additional constraint on the solar model atmospheres.

\subsubsection{An Example of F-state Interference Phenomena: Ba II $D_{2} 4554 \AA$}

The $\mathrm{Ba}$ II $\mathrm{D}_{2}$ line arises from the transition involving the upper $J=3 / 2$ and the lower $J=1 / 2$ fine structure levels (see Figure 4(a)). In the case of Sun, $18 \%$ of the total $\mathrm{Ba}$ abundance corresponds to odd isotopes and the remaining $82 \%$ to even isotopes. The odd isotopes of $\mathrm{Ba}$ have a nuclear spin $I_{s}=3 / 2$. Because of the coupling between the $J$ quantum number and the nuclear spin $I_{s}$, the upper and lower levels undergo hyperfine structure splitting (HFS). This gives rise to four upper and two lower $F$-states (see Figure 4(b)). Smitha et al. (2013b) demonstrate the necessity to account for PRD, the isotopic shifts and the quantum interference between the upper $F$-states to model the observed linear polarization profiles of the Ba II $\mathrm{D}_{2}$ line.

Figure 5 shows the theoretical fit to the observed $Q / I$ spectrum of the $\mathrm{Ba} I \mathrm{I} \mathrm{D}_{2}$ line. The observations were performed by Stenflo and his team in 2008 using the ZIMPOL-2 polarimeter at THEMIS. The data were recorded at the heliographic north pole with the slit placed parallel to the limb at $\mu=0.1$. To take into account the different isotopes of BaII, we used three atomic models corresponding to one even and two odd isotopes. The atomic model for the even isotope $\left({ }^{138} \mathrm{Ba}\right)$ is given by the five levels represented in Figure $4(\mathrm{a})$, while for the odd isotopes $\left({ }^{135} \mathrm{Ba}\right.$ and $\left.{ }^{137} \mathrm{Ba}\right)$ the model is extended to include the hyperfine splitting as represented in Figure $4(\mathrm{~b})$. The HFS of the ${ }^{2} \mathrm{P}_{1 / 2}$ level and the 
(a)

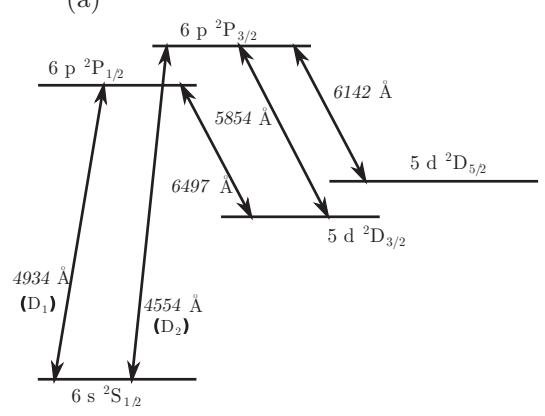

(b)

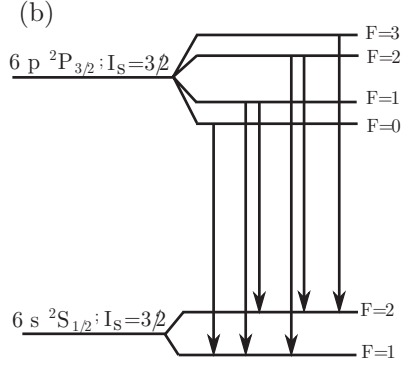

Figure 4. Ba II model atom for the even isotopes (a). For the odd isotopes the atomic model is modified by replacing two of the levels, ${ }^{2} \mathrm{P}_{3 / 2}$ and ${ }^{2} \mathrm{~S}_{1 / 2}$, with their hyperfine structure components (b). The energy levels are not drawn to scale.
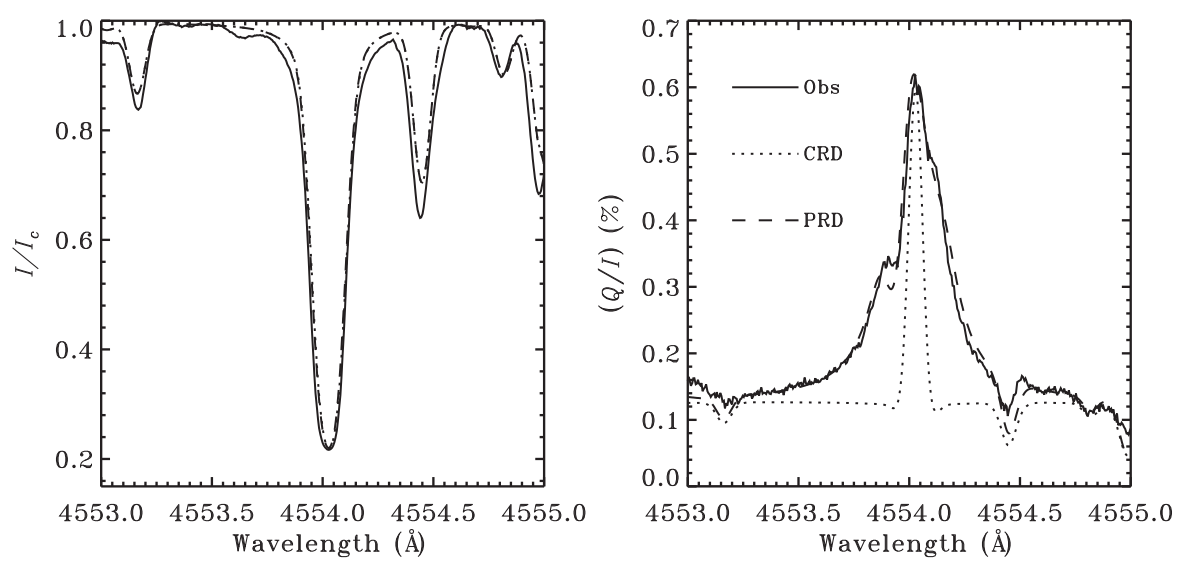

Figure 5. Fits to the observed $I / I_{c}$ (left panel) and $Q / I$ (right panel) profiles using the $\overline{\text { FALX }}$ model atmosphere, assuming PRD (dashed line) and CRD (dotted line). A $70 \mathrm{~m} \AA$ spectral smearing is applied to the theoretical profiles to simulate the observations. In the left panel $\left(I / I_{c}\right)$, the dashed and dotted lines are superposed on one another.

metastable levels are neglected. Further details can be found in Smitha et al. (2013b). The three atomic models of Ba II give us three different sets of physical quantities when used in the $\mathrm{RH}$ code to compute the intensity. These quantities are the line opacity, the line emissivity, the continuum absorption coefficient, the continuum emissivity, the continuum scattering coefficient, and the mean intensity. These three sets of quantities are then combined in the ratio of their respective isotopic abundances. The combined quantities are then used as inputs to the polarization code, where a two-level atom model is assumed for all three isotopes. The $F$-state angle-averaged PRD matrix derived in Smitha et al. (2012a) is used in the case of the odd isotope, while the usual two-level atom angle-averaged PRD matrix (see e.g., Bommier 1997a) is used for the even isotope. We assume the lower level to be infinitely sharp and unpolarized. The elastic collision rates that cause broadening $\left(\Gamma_{\mathrm{E}}\right)$ and depolarization $\left(D^{(2)}\right)$ are computed following Barklem \& O'Mara (1998) and Derouich (2008), respectively. In the computation of $D^{(2)}$, the collisional coupling between the ${ }^{2} \mathrm{P}_{1 / 2}$ and ${ }^{2} \mathrm{P}_{3 / 2}$ is included, while that between the ${ }^{2} \mathrm{P}_{3 / 2}$ and the metastable level ${ }^{2} \mathrm{D}_{5 / 2}$ is neglected.

The observed $Q / I$ spectrum of the $\mathrm{Ba}$ II $\mathrm{D}_{2}$ line shows a triple peak structure with wing polarization reaching the continuum far from the line center. It was shown by Stenflo 
(1997) that the central peak is due to the even isotopes of $\mathrm{Ba}$, and the two inconspicuous side peaks are due to the odd isotopes. The wing polarization can be reproduced only when PRD is taken into account (compare the dotted and dashed lines in Figure 5; see also Faurobert et al. 2009, who did radiative transfer calculations using only the even isotope of $\mathrm{Ba}$ ). The theoretical $Q / I$ spectrum shown in Figure 5 also includes the contribution from the spectrograph stray light, which is $4 \%$ of the continuum intensity. Furthermore, a micro-turbulent magnetic field with a strength of $2 \mathrm{G}$ was included to obtain a good $Q / I$ fit at the line center. From the figure it is clear that, although the intensity profile can be reproduced using either PRD or CRD, the $Q / I$ profile cannot be reproduced unless PRD is used. This demonstrates the importance of PRD in scattering polarization computations.

\section{Concluding Remarks}

Partial frequency redistribution is a realistic physical mechanism that can describe the formation of strong polarized lines correctly. In particular, it is responsible for the formation of the line-wing polarization. The mechanism of complete redistribution cannot be used to model the polarization in the line wings. In recent years, we have derived PRD matrices using the Kramers-Heisenberg approach, for quantum interference involving fine- and hyperfine-structure levels in a two-term atom. These PRD matrices are then applied to model the observed linear polarization in the Cr I triplet and the Ba II $\mathrm{D}_{2}$ line. We show that the PRD mechanism is essential to properly model the observed polarimetric data in spectral lines.

\section{Acknowledgements}

Financial support by the local organizing committee to attend the IAUS 305 is gratefully acknowledged. We thank Dr. M. Sampoorna for detailed discussions in the preparation of this manuscript. Thanks are also due to Drs. J. O. Stenflo, M. Bianda, R. Ramelli, L. S. Anusha, and Ms. H. N. Smitha for a fruitful collaboration. We acknowledge the use of the HYDRA cluster facility at the Indian Institute of Astrophysics for computing the results presented in this paper.

\section{References}

Anusha, L. S., Nagendra, K. N., Bianda, M., Stenflo, J. O., Holzreuter, R., Sampoorna, M., Frisch, H., Ramelli, R., \& Smitha, H. N. 2011, ApJ 737, 95

Barklem, P. S. \& O'Mara, B. J. 1998, MNRAS 300, 863

Bianda, M., Ramelli, R., Anusha, L. S., Stenflo, J. O., Nagendra, K. N., Holzreuter, R., Sampoorna, M., Frisch, H., \& Smitha, H. N. 2011, AछA 530, L13

Bommier, V. 1997a, A\&A 328, 706

Bommier, V. 1997b, A\&SA 328, 726

Bommier, V. 2014, in: K. N. Nagendra, J. O. Stenflo Z. Q. Qu, \& M. Sampoorna (eds.), Solar Polarization 7, ASP Conf. Series 489 (San Francisco: ASP), p. 195

Bommier, V. \& Stenflo, J. O. 1999, A\&A 350, 327

Carlin, E. S. \& Asensio Ramos A. 2015, ApJ 801, 16

Casini, R., Landi Degl'Innocenti, M., Manso Sainz, R., Landi Degl'Innocenti, E., \& Landolfi, M. 2014, ApJ 791, 94

Derouich, M. 2008, A\&A 481, 845

Faurobert, M., Derouich, M., Bommier, V., \& Arnaud, J. 2009, A\& A 493, 201

Fontenla, J. M., Avrett, E. H., \& Loeser, R. 1993, ApJ 406, 319

Holzreuter, R., Fluri, D. M., \& Stenflo, J. O. 2005, A\&\&A 434, 713 
Landi Degl'Innocenti, E., Landi Degl'Innocenti, M., \& Landolfi, M. 1997, in: N. Mein, \& S. SahalBréchot (eds.), Science with THÉMIS, Proc. Forum THÉMIS (Paris: Obs. Paris-Meudon), p. 59

Landi Degl'Innocenti, E. \& Landolfi, M. 2004, Polarization in spectral lines, Kluwer, Dordrecht Sampoorna, M. 2011, ApJ 731, 114

Sampoorna, M., Nagendra, K. N., \& Stenflo, J. O. 2007a, ApJ 663, 625

Sampoorna, M., Nagendra, K. N., \& Stenflo, J. O. 2007b, ApJ 670, 1485

Sampoorna, M., Nagendra, K. N., \& Stenflo, J. O. 2013, ApJ 770, 92

Smitha, H. N., Nagendra, K. N., Sampoorna, M., \& Stenflo, J. O. 2013a, JQSRT 115, 46

Smitha, H. N., Nagendra, K. N., Stenflo, J. O., Bianda, M., Sampoorna, M., Ramelli, R., \& Anusha, L. S. 2012b, A\& $A$ 541, A24

Smitha, H. N., Nagendra, K. N., \& Stenflo, Sampoorna, M. 2013b, ApJ 768, 163

Smitha, H. N., Sampoorna, M., Nagendra, K. N., \& Stenflo, J. O. 2011a, ApJ 733, 4

Smitha, H. N., Sowmya, K., Nagendra, K. N., Sampoorna, M., \& Stenflo, J. O. 2012a, ApJ 758 , 112

Sowmya, K., Nagendra, K. N., Sampoorna, M., \& Stenflo, J. O. 2014b, ApJ 793, 71

Sowmya, K., Nagendra, K. N., Stenflo, J. O., \& Sampoorna, M. 2014a, ApJ 786, 150

Stenflo, J. O. 1980, A\& A 84, 68

Stenflo, J. O. 1994, Solar magnetic fields - Polarized radiation diagnostics, Kluwer, Dordrecht

Stenflo, J. O. 1997, A\&A 324, 344

Stenflo, J. O. \& Keller, C. U. 1997, A\&\&A 321, 927

Trujillo Bueno, J. 2001, in: M. Sigwarth (ed.), Advanced Solar Polarimetry - Theory, Observation, and Instrumentation, ASP ASP Conf. Series 236 (San Francisco: ASP), p. 161

Trujillo Bueno, J., Landi Degl'Innocenti, E., Collados, M., Merenda, L., \& Manso Sainz, R. 2002, Nature 415, 403

Uitenbroek, H. 2001, ApJ 557, 389 